\title{
AN ACCOUNT
}

OF THE

\section{EXAMINATION OF A CYST CONTAINING} SEMINAL FLUID.

BY JAMES PAGET, F.R.C.S., LECTURER ON PHYSIOLOGY, AND WARDEN OF THE COLLEGIATE ESTABLISHMENT AT ST. BARTHOLOMEW'S HOBPITAL.

READ JUNE $25 T H, 1844$.

THE novel and interesting fact of the presence of spermatozoa in the fluids of certain hydroceles, which was communicated to the Society last year by Mr. Liston and Mr. Llovd, has not yet, I believe, been illustrated by examinations after death, and the conditions in which so strange a thing occurs have been hitherto only guessed at. I therefore beg to present to the Society an account of some observations which I have recently made, and which seem to me to throw some light upon the subject.

A middle-aged man was admitted into St. Bartholomew's Hospital, under Mr. Stanley, six months ago, with what was regarded a common hydrocele of the tunica vaginalis on the left side. This was tapped, and several ounces of a serous fluid, of the kind usually found in such hydroceles, were drawn off. The fluid was not particularly examined; the hydrocele was not injected; and, soon after, the man left the hospital. He returned with the hydrocele again full; and, besides, with very extensive ab- 
scesses in the perineum and fistulous openings into the urethra and bladder. With these he died, extremely emaciated, on Wednesday, June 5th; and the parts connected with the hydrocele were removed for examination.

The sac which had been tapped, and which, in its external appearance, even after its removal from the body, had all the characters of a common hydrocele, was found to be completely separate, and its cavity distinct from that of the tunica vaginalis. It was situated just above the testicle, and in front of the spermatic cord; it was of an elongated oval form, four inches in length, and held about six ounces of a bright light-yellow fluid, containing a small quantity of albumen, but no trace of either spermatozoa or any other organic particles. Its walls were very thin, and loosely connected with the adjacent parts ; they were composed of well-organized delicate fibrocellular tissue, and their internal surface was smooth, but not lined by any epithelium. The sac was closed on every side: it was separated from the tunica vaginalis by tissue like that of false membrane, layers of which formed several incomplete cysts, or spaces, containing a serous fluid like that in the sac itself.

The tunica vaginalis appeared healthy ; its cavity contained about three drachms of fluid, presenting the same external appearance as that in the sac, but containing numerous granules, together with some pale, round, granular corpuscles, very like the pale corpuscles of the blood, and, in a few instances, 
united by their edges into small groups like films of buffy coat. But there was in this fluid no more trace than in the others, of either spermatozoa, or any other constituent of the semen.

By the upper part of the epididymis, on its inner side, and attached to its surface, where on each side the tunica vaginalis is reflected from it, there was a globular cyst, about two-thirds of an inch in diameter, completely distinct from those already described, though almost surrounded by them. Its walls were thin, but opaque white ; they were composed of fibro-cellular tissue, with delicate pale filaments, intricately interwoven, and not so fully organized as that of the large sac. From its polished inner surface I scraped scales of an exceedingly delicate tessellated epithelium, composed of very pale, elongated, oval, and angular cells, united by their obscure edges, and having dark large nuclei of the same shape as themselves : they were much like the epithelium-cells of the tunica vaginalis itself, but even smaller than those of the blood-vessels. The contents of the cyst were two or three drachms of an opaque whitish fluid, in which there were numerous spermatozoa, dead and small, but well formed, and still more numerous granules, and large round granular spermatic globules. It contained no albumen coagulable by heat.

This cyst was closed on every side, and loosely connected to the adjacent parts, so that without cutting, and with very little force, it could be easily separated from them. The part of the surface of 
the epididymis to which it was attached was left, after its separation, perfectly smooth, and without the least appearance of a breach in the investing membrane, beneath which the fine convolutions of the seminal duct were seen, uninjured and undisturbed. It was as evident as it could be that the cavity of the cyst was completely isolated from every part of the seminal tubes.

If, with the aid of these observations, we endeavour to find an explanation of the occurrence of spermatozoa in the fluid of cysts connected with the testicle, we may suppose either that the fluid part of the semen has permeated from the seminal tubes into the cysts, and been further organized in them; or, that the cyst itself secretes a fluid in which the organic structures of the semen may be developed. Such a permeation is hardly possible ; for the fluid would have to pass not only through the vascular wall of the tubes, but through two or three more layers of vascular tissue, by all of which it would be absorbed rather than transmitted. The most probable explanation of these cases, therefore, seems to be, that certain cysts, seated near the organ which naturally secretes the materials for semen, may possess a power of secreting a similar fluid. And this explanation is in some measure supported by the analogy of those cysts which are found in the ovaries, and more rarely in other parts of the body, especially beneath hairy parts of the skin, and in which the ordinary products of the skin, such as epidermis, sebaceous matter, hair, \&c., are formed 
on the genuine cutaneous tissue* of their internal surface.

The opaque white fluid found in cysts by the testicles should, probably, be regarded, not as an ordinary serous or watery fluid containing spermatozoa, but as semen more or less perfectly formed. Such fluid, even when spermatozoa are not in it, presents the peculiar general appearance of diluted semen, and, unlike the serous fluids, contains little or no albumen; and, probably, though there may be no perfect spermatozoa, it may contain them imperfectly developed, or some of the other organic structures of the semen. In a body examined last winter, I found two cysts, one on the exterior and upper part of each epididymis, exactly like that just described as containing the spermatozoa, except that they were rather larger. Both of them were isolated and closed on every side; and they contained a thin, opaque-white fluid, in which, together with numerous glistening granules, I found several pale, round, granular corpuscles. In a few instances, several of these corpuscles were collected in small clusters, together with short and slender pale filaments, tapering at one end, exactly like the tails of spermatozoa. A few filaments of the same kind were scattered loosely in the fluid, and I found two which were slightly enlarged at one end, approaching more nearly to the form of complete

See Kohlrausch, in Müller's Archiv. 1843, Heft iv. p. 365 : whose observations I have verified in several specimens of ovarian cyots. 
A Cyst containing geminal fluid. 403

spermatozoa. I believe that all these filaments were ill-formed spermatozoa; and, if they were, this case is another example of cysts near the epididymis secreting seminal fluid, and affords evidence that such cysts, in different cases, possess the power of secreting the fluid in various degrees of perfection.

The existence of a large sac on the spermatic cord, unconnected with the tunica vaginalis, yet containing a fluid like that of common hydrocele (as in the case above related), suggests the possibility, that in the instances in which common hydroceles have been supposed to contain spermatozoa, the fluid has been drawn from a sac of this kind, and not from the cavity of the tunica vaginalis. It is, of course, not impossible that the tunica vaginalis should secrete a seminal fluid; but I think further evidence is necessary to prove that it ever does; for none of these cases have been examined by dissection, and the diagnosis during life is very uncertain. In the case which I have described, the fluid was supposed, even by our president, to be in the tunica vaginalis; and Mr. Liston speaks of the diagnosis between common hydroceles and large encysted hydroceles, as " frequently exceedingly difficult, if not impossible."*

To explain the occurrence of spermatozoa in the yellow albuminous fluids drawn from such cysts (or, possibly, from the tunica vaginalis), we may suppose

* Medico-Chirurgical Transactions, vol. xxvi. p. 218.

2 D 2 
that a part of the wall of the cyst secretes the materials for semen, while the rest secretes serum; a supposition which is supported by the analogy of the ovarian and similar cysts already mentioned. In these it is commonly only a part of their inner wall which has the structure, or forms the ordinary products, of skin; the rest has a simple structure, and produces, if anything, a simple serous fluid, and, as in these cysts, $I$ have found reason to believe that the skin, after existing and secreting for a certain time, may degenerate, cast off its hair and epidermis, and lose all its peculiar structures; so, probably, that which was once a seminal cyst, may cease to secrete semen, and produce only a serous fluid. 